\title{
Reflets
}

Revue ontaroise d'intervention sociale et communautaire

\section{L’épicerie-bus : un partenariat qui nourrit la communauté}

\section{Hélène Larochelle}

Volume 1, numéro 2, automne 1995

La santé communautaire en Ontario français : défis et espoirs

URI : https://id.erudit.org/iderudit/026091ar

DOI : https://doi.org/10.7202/026091ar

Aller au sommaire du numéro

Éditeur(s)

Reflets : Revue ontaroise d'intervention sociale et communautaire

ISSN

1203-4576 (imprimé)

1712-8498 (numérique)

Découvrir la revue

Citer cet article

Larochelle, H. (1995). L'épicerie-bus : un partenariat qui nourrit la communauté. Reflets, 1(2), 237-242. https://doi.org/10.7202/026091ar

Tous droits réservés (C) Reflets : Revue ontaroise d'intervention sociale et communautaire, 1995
Ce document est protégé par la loi sur le droit d'auteur. L'utilisation des services d'Érudit (y compris la reproduction) est assujettie à sa politique d'utilisation que vous pouvez consulter en ligne.

https://apropos.erudit.org/fr/usagers/politique-dutilisation/ 


\section{L'épicerie-bus : un partenariat qui nourrit la communauté}

\section{$H$ élène $L$ arochelle}

A gente de développement communautaire

C entre de santé communautaire du $\mathrm{C}$ entre-ville, 0 ttawa

Q ui d'entre nous n'a jamais eu à aider un parent âgé à faire son épicerie? Q ui peut prétendre «vieillir chez lui» en santé, s'il ne peut facilement se procurer la nourriture dont il a besoin pour se garder en santé?...

Tous les intervenants et intervenantes qui travaillent avec les aînés savent jusqu'à quel point leur bien-être peut dépendre de leur capacité de s'alimenter et d'acheter leur épicerie. Les aînés eux-mêmes parleront de leurs difficultés en ce domaine plusfacilement qu'ils n'admettront tout autre problème. Pour quiconque tente d'établir un lien avec eux, il est donc plus facile d'offrir un service qui réponde d'abord à des besoins primaires, comme un service de transport à l'épicerie, pour ensuite leur permettre de tisser graduellement des liens. Tout autre type d'intervention, s'il y a lieu, ne sera possible qu'au moment où la confiance sera établie et l'occasion, offerte et non imposée. C 'est dans cette optique que certains partenaires, dont le Centre de santé communautaire du C entre-ville d' $O$ ttawa et le centre pour les aînés $L$ es $B$ ons $C$ ompagnons, ont décidé de s'engager dansl'aventure de l'Épicerie-Bus.

Le Centre de santé communautaire (csc) du Centre-ville d'O ttawa offre à sa population, depuis plus de 25 ans, des programmes de santé communautaire; l'un d'eux, un programme des tinéaux aînés, sappelle sH IPs (Seniors' H ealth and Interest ProgramS). 
SHIPS est un programme de promotion de la santé, c'est-à-dire que nous travaillons avec les gens à promouvoir les conditions environnementales et sociales propices à une meilleure qualité de vie pour tous les membres de la collectivité.

L'une des stratégies privilégiées en promotion de la santé est celle du développement communautaire. J'aimerais partager avec vous une expérience de développement communautaire vécue à 0 ttawa, celle de la mise sur pied de l'Épicerie-Bus, ou l'histoire d'un partenariat qui nourrit la communauté.

C'est en tant qu'agente de développement communautaire pour SHIPS que j'ai été appelée à mettre sur pied, avec d'autres partenaires, le programme de l'É picerie-Bus.

Partant du principe que l'isolement des aînés est un des principaux obstacles à leur santé globale, et une des causes majeures du dépérissement de leur santé physique et mentale, un programme comme celui de l'Épicerie-Bus a été vu comme un moyen privilégié de combiner divers objectifs et de répondre à plusieurs besoins à la fois.

L'intérêt de ce programme réside dans son approche, soit un partenariat entre quatre types de partenaires: des aînés, un csc (C entre de santé communautaire du C entre-ville d'O ttawa) par le biais de son programme SHIPS, un centre pour aînés (Les B ons Compagnons) et l'entreprise privée, en l'occurrence, un magasin d'alimentation. Chacun des partenaires avait au départ des objectifs précis, et chacun souhaitait y trouver son profit.

À ce point, il est permis de se poser la question suivante: comment un simple service de transport à l'épicerie pouvait-il répondre aux objectifs de tant de gens? Q uelles étaient les attentes de chacun des partenaires?

Voyons d'abord le groupe des aînés: manifestement, la difficulté rencontrée par un grand nombre d'aînés à faire leurs achats d'épicerie justifie à elle seule la mise en place d'un programme susceptible de les aider. $0 \mathrm{n}$ rencontre peu de résistance à admettre ce besoin et le recrutement n'est pas difficile.

Le Programme de soutien à domicile du centre pour aînés visait, quant à lui, à assurer de l'aide et du transport pour per mettre 
aux aînés d'avoir plus facilement accès à la nourriture. Le soutien des aînés à domicile constitue l'essence de son mandat et constitue une belle façon de faire connaître les programmes et de recruter des bénévoles.

Pour sa part, SH IPS poursuivait plusieurs objectifs: utiliser une approche de développement communautaire pour créer entre les aînés, des réseaux d'amitié et d'entraide destinés à briser leur isolement; rendre accessible l'information au sujet des ressources communautaires et de santé et, au besoin, référer les ainés aux personnes appropriées; promouvoir les programmes d'éducation à la santé; et créer des conditions propices à l'action sociale, communautaire et politique des aînés.

Enfin, en ce qui concerne le magasin d'alimentation, on professait des objectifs économiqueset sociaux. La clientèle des aînés au centre-ville d'O ttawa est nombreuse et mérite qu'on s'en préoccupe.

Les trois partenaires visaient également à accroître leur visibilité dans la collectivité et auprès des aînés.

C e programme a donc été mis sur pied en 1991. Q uatre années après ses débuts, il répond toujours aux objectifs de ses parrains et constitue une réponse fort intéressante aux préoccupations de ses usagères et usagers.

$M$ ais qu'est-il exactement? Vu de l'extérieur, il ressemble à n'importe quel service de transport pour aller à l'épicerie, sauf qu'il s'adresse à des aînés vivant dans des logements privés, appartements ou maisons du centre-ville d'O ttawa, et qu'il est relié à un réseau de services communautaires et sociaux.

U ne fois par semaine, un autobus scolaire, payé par le magasin d'alimentation, les ainés inscrits au programme conduit afin qu'ils puissent faire leurs courses en compagnie d'amies et d'amis. En cours de route, les responsables du programme (à l'origine, une de SHIPS et l'autre, du centre pour aînés) feront en sorte que leur voyage à l'épicerie leur permette de rapporter à la maison bien plus que de l'épicerie.

À bord, et à la pause-café dans le magasin, on soulignera les anniversaires, on signera la carte de prompt rétablissement pour 
I'une ou l'autre, on recevra de l'information sur les services existants dans la communauté et on parlera de la dernière menace à son régime de pension. En un mot, on participera à la vie de la communauté par le biais d'un véhicule privilégié...

Et c'est en effet un programme bien particulier! O ù donc une personne âgée pourrait-elle à la fois prendre l'autobus à sa porte; entrer en contact chaleureux avec du personnel qui la connaît bien; entendre parler des services sociaux communautaires; renouer d'anciens liens et faire de nouvelles connaissances; se mêler à un groupe pour participer à des activités extra-murales; se trouver de l'aide pour faire son ménage; devenir bénévole pour un programme d'aide aux aînés; en apprendre davantage sur le stress et faire les liens entre nutrition et santé; rencontrer les professionnelles de la santé de SHIPS; s'inscrire à un atelier sur le sommeil (au centre de santé), ou à un rendez-vous avec la pédicure (du centre pour ainés); signer une pétition contre l'âgéisme des annonces publicitaires; s'organiser pour protester contre le nouvel horaire de l'autobus du quartier; parler de ses problèmes avec des amis (et en régler quelques-uns en cours de route); tout cela dans le cadre de sa sortie hebdomadaire pour aller faire son épicerie?

D epuisqu'elles se sont inscrites à ce programme, des personnes sont devenues des activistes en faveur des droits des personnes ainées, et elles sont de toutes les batailles; certaines ont retrouvé une voix qu'elles pensaient avoir perdue, d'autres ont découvert avec plaisir le pouvoir du groupe. Des voisines de palier depuis plus de dix ans, qui se saluaient vaguement dans la salle de lavage, jouent maintenant aux cartes ensemble et font les courses de I'une ou de l'autre en cas de maladie. Est-ce le temps du vaccin contre la grippe? O n recevra de l'information, on se verra encouragé à visiter son médecin de famille ou, le cas échéant, on pourra s'inscrire à une des cliniques tenues à cet effet. Le temps des élections permettra un échange animé d'opinions et on s'organisera pour se rendre ensemble à une des assemblées. O n évitera ainsi les pièges des sorties le soir et on en profitera pour remettre à sa place le faiseur de trouble, selon la plus belle tradition des anciennes cabales électorales. 
L'environnement protégé offert par le groupe permet à certaines octogénaires de sortir de leur logement, une fois la semaine, et de garder un contact avec leur milieu, ne serait-ce que du haut de leur banc d'autobus. $M$ ais cet environnement, tout protégé qu'il soit, va favoriser des prises en charge par certaines, lorsque surgissent des situations qui réclament des actions communes. Ainsi, à la suite de multiples plaintes des aînées au sujet de l'état des trottoirs enneigés et/ ou glacés de la ville, plusieurs ont répondu à l'invitation des membres d'un groupe d'action et se sont impliquées dans toutes lesétapes qui ont mené à la mise en place, par laVille d'O ttawa, d'un projet pilote destiné à améliorer l'entretien de ces dits trottoirs. C ette réussite n'aurait pas été possible sans la participation soutenue de plusieurs aînées qui ont été recrutées dans l'É picerie-Bus.

$M$ ettre le pied à bord de l'autobus, c'est ouvrir la porte à tout un monde d'information et de ressources. Si l'on veut, on peut avoir accès à toute la gamme des services et des programmes, ou I'on peut se faire référer à l'un ou l'autre des intervenants du réseau communautaire, social ou de santé. Pour un accès rapide à la travailleuse sociale ou à l'infirmière de SHIPS, à la gestionnaire de cas des Soins à domicile, sans oublier l'aide juridique ou la date de la réunion communautaire sur la sécurité, l'Épicerie-Bus est pour l'instant un préarseur du «guichet unique» pour ses habitués: tous savent qu'ils trouveront là une piste de solution pour répondre à leurs préoccupations.

Un tel programme est exigeant pour ses responsables et ne s'improvise pas du jour au lendemain; il faut le développer à l'image de ses usagers, avec leur aide, et l'entretenir scrupuleusement. La personne en charge du programme, responsable du roulement et du suivi, doit toujours pouvoir être disponible et à l'écoute. Ses bénévoles doivent être formées et leur fidélité est essentielle pour établir des liens avec les aînés. En effet, comment pourrait-on reconnaître la personne dont l'état de santé décline, si on ne la voit pas de façon régulière?

Avant de conclure, j'aimerais vous entretenir du fait français dans ce programme. II y a deux aspects intéressants à la question: 
le programme lui-même, et ses répercussions. D'abord en tant que francophone à l'origine du programme, j'ai pu imprimer une marque bilingue dès le début: la majorité des aînés du centreville parlent anglais, mais pour ce qui se rapporte à toute la publicité pour l'Épicerie-Bus, à toute l'information diffusée et à toutes les interactions survenues dans le cadre de ce programme, j'ai toujours pu communiquer en français avec les membres. Je n'insisterai pas ici sur l'importance de pouvoir être malade ou de parler de ses préoccupations dans sa langue maternelle. Q u'il suffise de dire que nous avons toujours essayé de respecter cette exigence, même avec des aînés bilingues pour la plupart.

Au bout de deux ans de développement et d'opération, le programme étant en fort bonne posture et ayant atteint plusieurs de ses objectifs de départ, la gestion totale du projet est revenue à la responsable du Programme de soutien à domicile du centre pour aînés Les Bons Compagnons, une anglophone. À son propre regret, l'usage du français a décliné à bord de l'Épicerie-Bus, mais les autres contacts sont restés les mêmes. SH IPS est demeuré associé au programme, mais de plus loin.

U ne bonne nouvelle, c'est que ce programme a été à l'origine de plusieurs autres à travers la ville et à travers le $C$ anada. D es présentations à des conférences à Edmonton et à $M$ ontréal ont soulevé suffisamment d'intérêt pour donner naissance à d'autres expériences à St-Jean deTerre- $\mathrm{N}$ euve, à M ontréal et en $\mathrm{N}$ ouvelle-E cosse. C ertains de ces projets sont en français, comme à 0 ttawa avec Le Service d'entraide communautaire, et d'autres programmes de soutien à domicile ont repris le modèle bilingue, selon la clientèle. $M$ ais partout, ce qui fait la différence d'avec les autresservices du genre, c'est que l'É picerie-Bussoit un programme intégré, et qu'il s'avère le premier pas vers une meilleure qualité de vie globale pour les personnes aînées qui l'utilisent. 